\title{
Gestión de la demanda en las redes inteligentes. Perspectiva y control desde el usuario y la distribuidora
}

\author{
Sergio N. Bragagnolo ${ }^{1}$, Jorge C. Vaschetti ${ }^{1}$, Fernando Magnago ${ }^{2}$ y Juan C. Gomez-Targarona ${ }^{2}$ \\ (1) GECAP - Facultad Regional Córdoba - Universidad Tecnológica Nacional, Maestro M. Lopez esq. Cruz Roja \\ Argentina, Córdoba, Córdoba, Argentina (correo-e: sbragagnolo91@gmail.com; jvaschetti@gmail.com) \\ (2) Facultad de Ingeniería, Universidad Nacional de Rio Cuarto, Rio Cuarto, Córdoba, Argentina \\ (correo-e: fernando.magnago@gmail.com; jcgomez@ing.unrc.edu.ar)
}

Recibido Dic. 4, 2019; Aceptado Ene. 30, 2020; Versión final Mar. 3, 2020, Publicado Jun. 2020

\begin{abstract}
Resumen
El objetivo de este artículo es desarrollar el concepto de gestión de la demanda en relación a los tipos de control existentes y presentar un ejemplo simple de optimización de la demanda para evaluar las potencialidades de un control híbrido. Se presenta una breve introducción, detallando el conocimiento actual en gestión de demanda, se desarrolla el concepto de control directo e indirecto sobre la demanda, se identifica, y se elabora una tabla con las cargas residenciales. Se modelan dos residencias que podrán modificar su carga ante un esquema de precios dados y un objetivo de no concentración de la demanda fijados por la distribuidora, obteniendo una correcta optimización y dispersando las cargas de diferente forma en la segunda ronda. Se concluye que una intervención de la distribuidora puede mejorar la distribución de la demanda por la aleatoriedad del algoritmo genético sin comprometer la privacidad del usuario.
\end{abstract}

Palabras clave: gestión de la demanda; respuesta a la demanda; redes inteligentes; optimización; algoritmo genético

\section{Demand management in smart networks. Perspective and control of users and suppliers}

\begin{abstract}
The article aims is to develop the demand side management concept in relation to existing control types. It also aims to providing a simple example of demand optimization to evaluate the potentialities of a hybrid control. A brief introduction is presented detailing the state of the art. Then, the direct and indirect control over demand was discussed, the types of loads were described, and a table with the residential loads was prepared. Two residences were modeled, which could modify their load before a given price scheme and a nonconcentration objective set by the distributor. This resulted in obtaining a correct optimization and distributing of the loads in a different way in the second round. It is concluded that an intervention of the distributor can improve the distribution of the demand by the randomness of the genetic algorithm without compromising user privacy.
\end{abstract}

Keywords: demand side management; demand response; smart grids; optimization; genetic algorithm 


\section{INTRODUCCIÓN}

El incremento de Generación Distribuida (GD) proveniente de fuentes de energías renovables y la incorporación de nuevas tecnologías, plantean nuevos desafíos donde los sistemas de inteligencia, control y operación de las redes se propagan sobre los de distribución. Esto permite al operador gestionar la demanda. Además, surge el concepto de prosumidor que demanda energía de la red y vierte energía sobre ella por poseer GD. Por ello, se están desarrollando sistemas para operar y controlar la GD. También se incorporan nuevas tecnologías del lado de la demanda, que la hacen más fluctuante y con mayores picos, aumentando las perturbaciones en las redes eléctricas. Por estos motivos, muchos investigadores consideran que las redes inteligentes permitirán la gestión de la demanda y precisarán de programas de Respuesta a la Demanda (RD) para disminuir la sobredimensión de la red y sus costos asociados, mientras se logra mayor confiabilidad (Blaauwbroek et al., 2017; Kassakian et al., 2011; Restrepo et al., 2018; Seema et al., 2016; Zhu et al., 2015). Bajo estas premisas se han propuesto algoritmos para la gestión residencial de la demanda.

La siguiente literatura analizada utiliza métodos tradicionales: 1) Melhem et al. (2018) utiliza el método de optimización lineal entera mixta combinado con el algoritmo voraz, resaltando un método híbrido matemáticoheurístico. El algoritmo voraz genera todas las soluciones de óptimo local en cada último periodo de optimización y obtiene la solución del óptimo global, en un tiempo aceptable de computación. Concluye que el algoritmo mixto llega a la solución más rápido que el método de optimización lineal entera mixta (MILP por sus siglas en ingles) y el costo es apenas mayor, dando el resultado esperado. 2) Janocha et al. (2016) utiliza un MILP para gestionar la demanda y el objetivo es optimizar la función costo, sin tener en cuenta el confort. Los autores de ese trabajo acotan, como desventaja, que el optimizador prioriza la solución más barata. 3) En Huang et al. (2016) realizan una revisión bibliográfica y concluyen que la mayoría de los artículos fueron realizados con optimización uni-objetivo por lo tanto proponen un problema multi-objetivo que incluye el costo y el confort del usuario y utilizan el método de optimización no lineal entera mixta. El problema es convertido en uni-objetivo al tratar el confort como un costo asociado y agregarlo a la función objetivo con un factor de ponderación. 4) F. Wang et al. (2018) presentan una optimización multi-objetivo que considera costo y confort utilizando la herramienta YALMIP de Matlab. 5) En Tan et al. (2016) es considerado un método de optimización lineal con flexibilidad de programación de la carga. 6) Finalmente Duman et al. (2018) propone un MILP para reducir el costo considerando que la potencia en horario pico no exceda la fijada por la distribuidora.

Los siguientes artículos revisados utilizan métodos metaheurísticos: 1) En Vidal et al. (2014) se optó por un algoritmo evolutivo al tener que trabajar con varios tipos de cargas de características de consumo diferentes propagadas a través del tiempo. Debido a la naturaleza de las cargas los autores consideraron que la programación lineal o la dinámica no eran apropiadas, además el algoritmo evolutivo permite cierta flexibilidad en el modelo y la priorización de cargas. En el modelado consideran un área residencial y la optimización de la curva de demanda del total de los usuarios pertenecientes a esa área. 2) Y. Wang et al. (2017) propone un algoritmo de optimización por enjambre de partículas (PSO por sus siglas en inglés) mejorado y comparan el PSO con la programación dinámica obteniendo menor costo y menor tiempo de ejecución. 3) Gupta et al. (2016) también propone un PSO justificando su elección de la misma manera que Vidal et al. (2014). Aquí el algoritmo es aplicado a la demanda de un área residencial para minimizar el pico de consumo y optimizar el costo. 4) Rahate y Kinhekar (2017) usan el PSO para optimizar la demanda de 200 usuarios y la distribuidora, que conoce las cargas controlables, realiza la optimización. Justifican su elección por la capacidad de este algoritmo para manejar un gran número de cargas frente a los métodos tradicionales. 5) Un algoritmo de colonia de hormigas es utilizado en Silva et al. (2015) y comparado con un algoritmo genético, para los casos propuestos el algoritmo propuesto mostró mejor eficiencia. 6) Un Algoritmo Hibrido Genético-Taguchi fue desarrollado para gestionar la demanda de una residencia que incluye batería y generación fotovoltaica en Lin y Chen (2016) y es comparado con un algoritmo genético para demostrar su eficiencia. 7) En Zafar et al. (2017) se propusieron tres algoritmos diferentes para la optimización de la demanda. Ellos son: Algoritmo de búsqueda harmónica, Algoritmo de optimización mediante forrajeo de bacterias y un algoritmo evolutivo mejorado, concluyendo que el primero es el más efectivo al reducir los costos. 8) Jayadev y Swarup (2013) propusieron un algoritmo genético para optimizar la demanda, considerando generación fotovoltaica y la inconveniencia que genera desplazar las cargas. 9) Pavithra y Esther (2017) usan un algoritmo genético para gestionar la demanda de diferentes tipos de usuarios.

De la literatura analizada se concluye que los trabajos que utilizaron algoritmos tradicionales optimizan la demanda de un usuario ante un esquema de precios dados, por medio de un enfoque de control indirecto. Mientras que los algoritmos heurísticos se usaron para cargas más complejas u optimización de un gran número de usuarios. Además, para optimizar un gran número de usuarios se requiere un control directo sobre las cargas interrumpibles de los consumidores. Como resumen del análisis se concluye que: 1) la literatura analizada para la optimización de una sola unidad residencial no considera los peligros de nuevos picos debido a la concentración de cargas en un horario de bajo costo; 2) los artículos que abarcan un gran número 
de consumidores tienen de objetivo la no producción de nuevos picos en la red, pero precisan de un control directo sobre los usuarios. Al considerar que se abordan usuarios residenciales, el control directo es de difícil aplicación al afectar la privacidad de los usuarios. Por estas razones en este artículo primero se profundiza sobre los conceptos de control directo e indirecto y luego se efectúa una optimización de dos unidades residenciales, realizada por los usuarios, pero con intervención de la distribuidora para evitar la concentración de la demanda en el horario de menor precio. El artículo se organiza de la siguiente forma: primero se define la gestión de la demanda, los tipos de controles existentes y se clasifican las cargas. Luego se presenta un ejemplo de gestión de demanda ante un control hibrido y finalmente se elaboran las conclusiones teniendo en cuenta las potencialidades de gestionar la demanda y las nuevas líneas de investigación con sus beneficios esperados.

\section{EL MODELO}

El modelo se presenta en tres subsecciones: en la primera se describe que es la gestión de la demanda, en la segunda se definen los tipos de control y en la última se clasifican las cargas y se elabora una tabla con las cargas que podrá tener cada usuario.

\section{Gestión de la demanda}

La gestión de la demanda consiste en controlar las cargas para operar el sistema con mayor eficiencia y sustentabilidad (Vidal et al., 2014). Ésta permite: usar varios criterios de optimización relacionados a la curva de demanda, mayor aprovechamiento de las energías renovables, menor cantidad de potencia a transmitirse en la red y mayores beneficios económicos (Vidal et al., 2014). Por medio de programas puede alterarse el patrón de consumo de los usuarios para modificar sus requerimientos de potencia, provocando cambios en la curva de demanda. En este sentido, la gestión de la demanda es un enfoque activo que permite dos grandes categorías de acciones: Reducción y Desplazamiento del consumo (Belhaiza y Baroudi, 2014). En la Figura 1 se muestran diferentes acciones que se pueden realizar en la demanda.
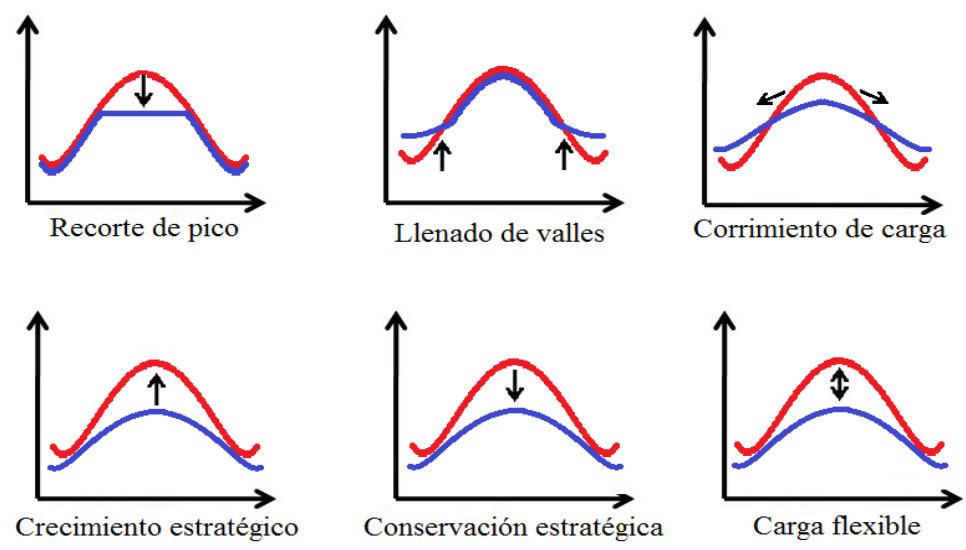

Fig. 1: Estrategias clásicas usadas para la DSM (Vidal et al., 2014).

Uno de los principales objetivos de la gestión de la demanda (DSM, por sus siglas en ingles) es disminuir los picos de demanda del sistema, para evitar nuevas inversiones al no necesitar aumentar la capacidad de generación, transmisión y distribución (Vidal et al., 2014). Estos tipos de programas pueden ejercer un control directo sobre la carga o un control indirecto. En el control indirecto la distribuidora no puede modificar la demanda, entonces tiene que utilizar herramientas, como el esquema tarifario, para influir en la demanda. La distribuidora necesitaría interactuar con el usuario para lograr el perfil de demanda deseado. En el control directo la distribuidora puede realizar modificaciones sobre la demanda ya sea de forma directa o a través de ordenes de desconexión.

Desde el punto de vista del usuario, se origina el paradigma emergente del consumidor-operador de micro redes inteligente (MG, por sus siglas en ingles). Este nuevo usuario es un cliente residencial o comercial que posee GD y puede gestionar su demanda. Por este motivo, posee la clave de las funcionalidades de la MG dentro de sus limitaciones económicas, técnicas y sociales. Con una alta penetración de GD en la red de distribución, este usuario tiene un rol significativo al administrar el uso de la energía eléctrica en el extremo de la distribución, mediante la elección de consumir la energía que produce o venderla en la red y la gestión de su demanda. Ante esto deben establecerse reglas comunes que permitan integrar los usuarios a la red y establecer contratos entre las distribuidoras y los usuarios que, con la inclusión del consumidor-operador, reduzca el impacto que provocaría una alta y descoordinada penetración de GD (Suryanarayanan et al., 2010). 
Desde el punto de vista de la distribuidora, el avance de la inteligencia sobre las MG, permite conocer en tiempo real lo que sucede, realizar estimaciones de estado (Blaauwbroek et al., 2017), establecer precios de la energía eléctrica en tiempo real y/o emitir órdenes dirigidas al usuario. Esto permite desplazar el consumo, prever fallas o, en caso de que ocurran, realizar acciones que restauraren el sistema rápidamente y gestionar la GD.

\section{Tipos de Control}

Con la implementación de esquemas de gestión de la demanda, de medidores inteligentes y de canales de comunicación, son necesarios mecanismos de incentivo para que los usuarios desplacen su consumo a periodos en que la red este menos cargada. Es usual que se elaboren diferentes cuadros tarifarios como mecanismo de incentivo que permitan pagar menos por la energía en los horarios de menor consumo (Tatarenko y GarciaMoreno, 2014). En este sentido, hay dos tipos de estrategias principales de DSM: Control directo de la carga (CD) o Control indirecto de la carga (Cl), utilizando programas basados en el precio de la energía (Tatarenko y Garcia-Moreno, 2014). A pesar de que los métodos basados en el precio de la energía requieren menor infraestructura de control y comunicación, es el CD el que evita mejor los picos críticos de demanda y además provee la regulación del servicio (Ej. La regulación de frecuencia para manejar la imprevisibilidad de la energía renovable) (Erdinc et al., 2016). En la figura 2 se observa un resumen de la clasificación de los tipos de control.

$$
\begin{aligned}
& \text { Tipos de }- \text { Control Directo } \\
& \text { Control } \\
& \rightarrow \text { Control Indirecto }\left\{\begin{array}{l}
\text { Permite el despacho de la demanda. } \\
\text { Elabora requerimientos u órdenes a cumplir por los usuarios. } \\
\text { Puede implicar duras restricciones al usuario. }
\end{array}\right. \\
& \left\{\begin{array}{l}
\text { Establece mecanismos tarifarios como time of use, real peak } \\
\text { pricing o critical peak pricing } \\
\text { Necesita de algoritmos de optimización para obtener el menor } \\
\text { costo }
\end{array}\right.
\end{aligned}
$$

Fig. 2: Clasificación de los tipos de control.

En el Cl el flujo de información está compuesto por señales de precio transmitidas desde el distribuidor al usuario. Presenta un enfoque basado en las señales de precios, la venta y la optimización del consumo en el usuario utilizando algoritmos computacionales para conseguir el menor costo. El Cl es usado en los trabajos que abordan la RD. El sistema de precios elegido es monitoreado constantemente para evitar un desplazamiento de los picos de consumo (Dethlefs et al., 2014). Dentro de los diferentes esquemas de precios se encuentran: tiempo de uso, precio en tiempo real o precio de pico crítico. En el $\mathrm{Cl}$ el consumidor gestiona sus equipos (Kassakian et al., 2011). Las investigaciones realizadas respecto al $\mathrm{Cl}$ pueden enfocarse en la curva de demanda del usuario $\mathrm{o}$ sobre la curva de demanda resultante en la micro red. En el primero se usan algoritmos de optimización para abaratar el uso de la energía eléctrica optimizando la curva de demanda. En el segundo, los trabajos analizados usan teoría de juegos, donde la red propone un sistema de precios y cada usuario elabora la estrategia de consumo que reduzca su costo energético.

Además, la red conoce la demanda y/o generación del usuario y si puede cumplir los requerimientos solicitados. EI CD se centra en el uso de protocolos y señales. Un ejemplo de CD es un distribuidor u operador con completo control sobre las cargas, realizando un despacho de la demanda. Aquí el sistema responde de una manera determinada ante diferentes señales. Un problema en el CD es que puede implicar duras restricciones al consumidor. Además, el operador requiere información detallada sobre la carga a controlar para anticipar los efectos del control. En este enfoque el control está distribuido. En la Figura 3 se observa un esquema simplificado del flujo de potencia e información, válido para Cl y CD. El esquema se elaboró a partir de Seema et al. (2016) y del análisis bibliográfico realizado. El flujo de potencia es igual en ambos y el flujo de información tiene diferentes atributos según el control (Cl o $\mathrm{CD}$ ).

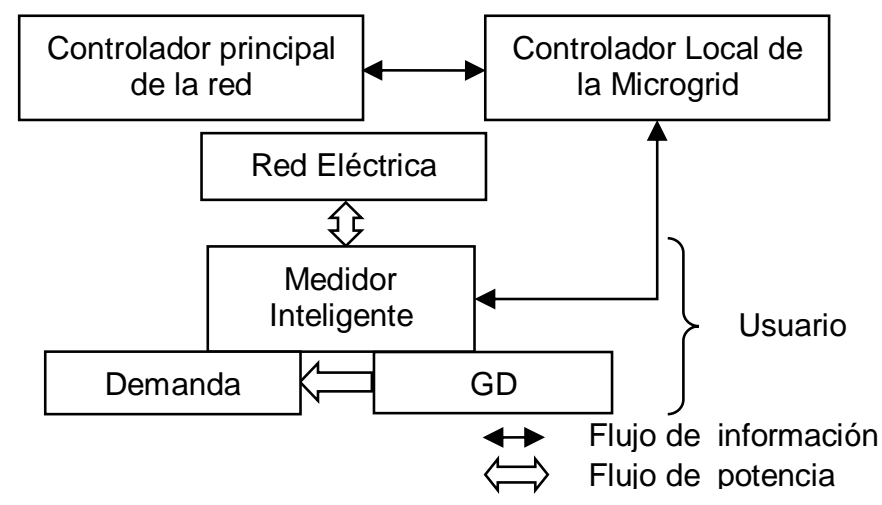

Fig. 3: Esquema simplificado de la red inteligente. 
Un CD aumenta la confiabilidad y la respuesta a tiempo de la red ante condiciones normales de funcionamiento (despacho de demanda, despacho de generación distribuida, regulación de voltaje, regulación de frecuencia), y bajo condiciones anormales cómo picos de demanda pronunciados o fallas en la red. Debe tenerse en cuenta que la operación y control de la red no debe comprometer el confort del usuario. EI CD es realizado sobre cada prosumidor incorporado a la MG interactuando la red con cada unidad residencial, por eso es un control distribuido sobre la red. La Figura 4 presenta el diagrama de un CD. Se elaboró en base a Shao et al. (2011) y la revisión bibliográfica realizada. Ante la necesidad de despacho de demanda, la red analiza la información recopilada por los Sistemas de Gestión Central Residencial (SGCR) y por su propio sistema de medición y emite órdenes de diferentes niveles de prioridad a ser ejecutadas por cada SGCR. La ventaja para la MG, y para los prosumidores, es disponer de un autómata que opere la demanda y optimice el sistema por medio de la interacción entre los SGCR y la red.

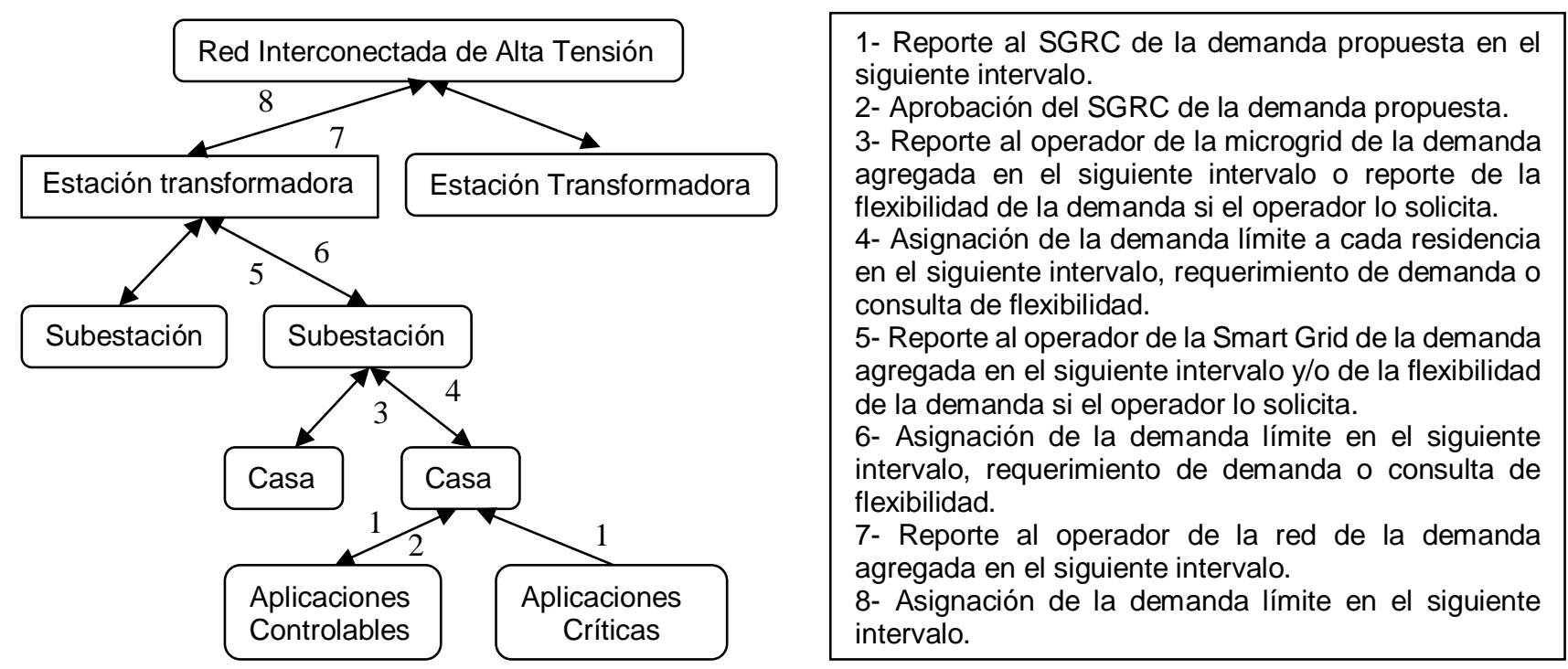

Fig. 4: Diagrama de Control Directo.

Puede pensarse un sistema hibrido de control, que en operaciones normales se comporta como un $\mathrm{Cl}$ a través de un sistema tarifario definido en tiempo real, con predicción a 24 horas, y para condiciones anormales de funcionamiento será un $\mathrm{CD}$, donde el operador solicita al usuario una reducción de consumo en base a la carga disponible informada previamente por el usuario.

Un ejemplo de cómo la integración usuario-red funcionará se observa ante un desequilibrio entre la oferta y la demanda que puede provocar cortes eléctricos o caídas de voltaje y reducir la cantidad de equipos conectados a la red. Ante esto, en una red integrada, la distribuidora emitirá una orden a cada usuario para que desconecte sus cargas no prioritarias, evitando una interrupción grave del servicio. Para que la integración exista, debe haber módulos de medición inteligentes en distribución que monitoreen en tiempo real la MG y permitan realizar estimaciones de estado. Una ventaja para el usuario es que puede programar su SGCR indicando cargas prioritarias, entonces ante un evento de desequilibro en la red, el SGCR, al llegar la orden de la distribuidora desconectará las cargas de menor prioridad. Esto permite que ante una falla, donde la MG quede aislada del sistema, las casas conectadas mantegan sus cargas esenciales y desconecten las de menor prioridad en forma automática. Esto provee una interesante solución a los cortes eléctricos (Seema et al., 2016). A partir de este ejemplo se observa que el distribuidor necesita comunicarse con las unidades residenciales para conocer las capacidades de los usuarios pero debe considerarse la privacidad de cada usuario. Si se piensa en usuarios celosos de su privacidad, es imposible que la red conozca con exactitud la demanda en juego de cada usuario, así como el volumen que puede interrumpirse o reducirse ante un pico de consumo. Se puede establecer un control híbrido con determinadas condiciones y reglas que le permitan al operador conocer el comportamiento de los usuarios. De esta forma la red puede estimar la reducción o incremento de la demanda ante un evento dado. En este trabajo se presenta un $\mathrm{Cl}$ híbrido que permite evaluar la utilidad de algoritmos aleatorios, como el genético, y la interacción usuario-distribuidor para aplanar la curva de demanda y evitar nuevos picos.

\section{Clasificación de las cargas}

Los aparatos y equipos de las unidades residenciales pueden clasificarse de acuerdo a sus características de consumo: 1) Cargas desplazables (D): son aquellas que pueden ser desplazadas desde el momento que se produce el pico de demanda a algún otro momento deseado o de valle de demanda. Como ejemplo de este tipo se encuentran los lavarropas, sistemas de almacenamiento de energía térmica, etc. El usuario puede posponer 
o interrumpir el uso de la carga sin afectar su calidad de vida, siempre que las operaciones se terminen dentro de un período de tiempo acorde y produzcan beneficios financieros; 2) Cargas interrumpibles (I): son aquellas que pueden ser interrumpidas momentáneamente. Puede ser necesaria su compensación al terminar el evento de $\mathrm{RD}$. Se puede usar un aire acondicionado (AC) como carga interrumpible al ser posible su interrupción en un intervalo predefinido, aunque finalizado el intervalo debe ser encendido para cumplir con la especificación de confort del usuario; 3) Cargas ajustables (A): son aquellas que la demanda de potencia es una variable continua y puede ser controlada por el sistema. Dentro de este tipo se puede ubicar el AC tipo inverter; 4) Cargas críticas (CC): son aquellas que no pueden operarse; como una heladera que permanece encendida todo el día. Las operaciones de estas aplicaciones son dominadas estrictamente por el confort y la necesidad, por lo tanto, su interrupción atenta contra la calidad de vida del usuario (Bian et al., 2015; Huang et al., 2016; Zhu et al., 2015).

Efectuando un análisis de las cargas que pueden existir en una unidad residencial, del tratamiento elaborado en la literatura revisada y de la información de consumos brindadas por el Instituto Nacional de Tecnología Industrial (INTI, 2019) y el Ente Nacional Regulador de la Electricidad (ENRE, 2019), se elaboró la Tabla 1.

Tabla 1: Clasificación de los electrodomésticos

\begin{tabular}{|c|c|c|c|c|}
\hline \multirow{2}{*}{ Tipo } & \multirow{2}{*}{ Clasificación } & \multicolumn{3}{|c|}{ Cargas residenciales } \\
\hline & & Electrodoméstico & Potencia [W] & Energía [Wh] \\
\hline CB & $\mathrm{CC}$ & Heladera con freezer & 195 & 98 \\
\hline CB & $\mathrm{CC}$ & Módem - Router & 10 & 10 \\
\hline $\mathrm{CB}$ & $\mathrm{CC}$ & Pava Eléctrica & 2000 & 2000 \\
\hline CB & $\mathrm{D}$ & Plancha & 1000 & 600 \\
\hline CB & $\mathrm{CC}$ & Televisor LCD & 100 & 100 \\
\hline CB & $\mathrm{CC}$ & Caja de TV por cable & 43,4 & 43,4 \\
\hline $\mathrm{CB}$ & $\mathrm{CC}$ & Luminarias LED & 8,5 & 8,5 \\
\hline CB & $\mathrm{CC}$ & Notebook & 60 & 60 \\
\hline CB & I & Ventilador Común & 90 & 90 \\
\hline CB & $\mathrm{CC}$ & Cargador de teléfono & 14 & 14 \\
\hline $\mathrm{CB}$ & $D$ & Lavarropas automático & 520 & 182 \\
\hline $\mathrm{CO}$ & $\mathrm{CC}$ & Computadora & 300 & 250 \\
\hline $\mathrm{CO}$ & $\mathrm{CC}$ & Horno microondas & 800 & 640 \\
\hline $\mathrm{CO}$ & $\mathrm{CC}$ & Equipo de sonido & 100 & 100 \\
\hline $\mathrm{CO}$ & $I-A$ & Aire acondicionado & 1350 & 1013 \\
\hline $\mathrm{CO}$ & I & Estufa de cuarzo & 1200 & 1200 \\
\hline $\mathrm{CO}$ & I & Ventilador de Techo & 60 & 60 \\
\hline $\mathrm{CO}$ & $\mathrm{CC}$ & Horno Eléctrico & 1300 & 1300 \\
\hline $\mathrm{CO}$ & $\mathrm{CC}$ & Licuadora & 300 & 300 \\
\hline $\mathrm{CO}$ & $\mathrm{CC}$ & Secador de Pelo & 500 & 400 \\
\hline $\mathrm{CO}$ & 1 & Calefón eléctrico & 2000 & 2000 \\
\hline $\mathrm{CO}$ & $\mathrm{CC}$ & Freezer & 180 & 90 \\
\hline $\mathrm{CO}$ & D & Aspiradora & 750 & 675 \\
\hline $\mathrm{CO}$ & $\mathrm{CC}$ & Cafetera & 900 & 720 \\
\hline $\mathrm{CO}$ & $\mathrm{CC}$ & Lavavajillas & 1500 & 1050 \\
\hline $\mathrm{CO}$ & $\mathrm{CC}$ & Plancha de pelo & 40 & 40 \\
\hline
\end{tabular}

Para modelar el comportamiento de los usuarios, se emplea como base los electrodomésticos de la Tabla 1. Algunos se consideran básicos (CB) y otros opcionales (CO). Entonces los básicos son asignados a todos los usuarios y los opcionales son asignados de forma aleatoria. Se considera que cada usuario tendrá asignado un televisor y podría tener asignado un segundo televisor. Luego se modelan dos usuarios siguiendo conductas de consumo observadas en curvas reales obtenidas de la base de datos ejemplo. Una vez determinadas las cargas correspondientes a cada usuario, se define la curva de demanda diaria (Celiz et al., 2018) 


\section{RESULTADOS Y DISCUSIÓN}

En este apartado se presenta un control indirecto híbrido con comunicación con la red y un sistema tarifario definido. A partir de la Tabla 1 se modelan dos usuarios de la red con una curva de demanda típica. Luego se simulan sus comportamientos ante las condiciones fijadas.

El operador informa el esquema tarifario a los usuarios, éstos determinan sus estrategias de consumo y se la envían al operador indicando las cargas flexibles. Se modelaron usuarios con 28 cargas cada uno y una sola carga flexible que será usada tres veces en el día, obteniendo la matriz A.

$A=\left[\begin{array}{ccc}a_{1,1} & \ldots & a_{1,28} \\ \vdots & \ddots & \vdots \\ \vdots & \ddots & \vdots \\ a_{24,1} & \ldots & a_{24,28}\end{array}\right]$ con $A \in R^{24 \times 28}$

El elemento aij de A es el estado de la carga j, que será 1 o 0 según este apagada o encendida, en el intervalo de tiempo i.

$$
E=\left[\begin{array}{c}
E_{1} \\
\vdots \\
E_{28}
\end{array}\right] \operatorname{con} E \in R^{28 \times 1}
$$

El elemento ei del vector columna E representa la energía consumida por la carga i.

Multiplicando A por E se obtiene la ecuación 3.

$$
X=A \cdot E \operatorname{con} X \in R^{24 \times 1}
$$

El vector $X$ es la estrategia de consumo de cada usuario, donde el elemento $x_{i}$ es la energía consumida por todas las cargas encendidas en el intervalo de tiempo i.

El operador propone un sistema tarifario $\mathrm{P}$ con un precio definido para cada hora.

$$
P=\left(p_{1}, p_{2}, \ldots, p_{24}\right) \text { con } P \in R^{24 \times 1}
$$

Como resultado de multiplicar (3) y (4) se obtiene un escalar que representa el costo asociado a la energía consumida por cada usuario en el día.

$$
C=P^{\prime} X \text { con } C \in R
$$

Para optimizar el costo de cada usuario se utiliza un algoritmo genético (Gestal et al., 2010). En las Figuras 5 y 6 se muestra la lógica de los algoritmos utilizados.

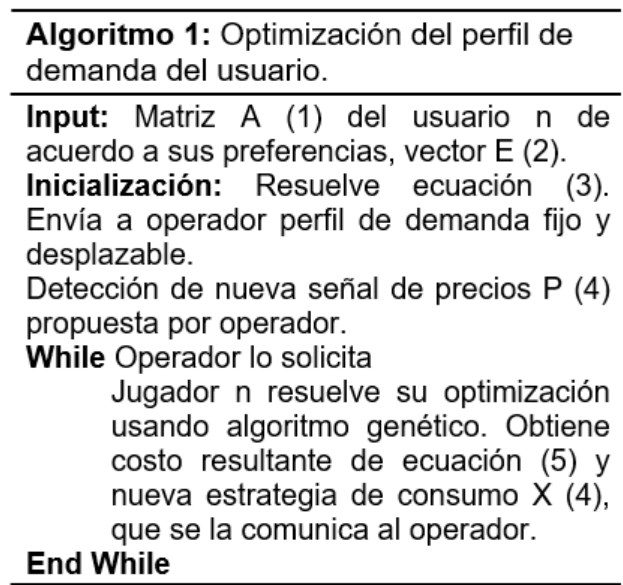

Fig. 5: Optimización del perfil de demanda

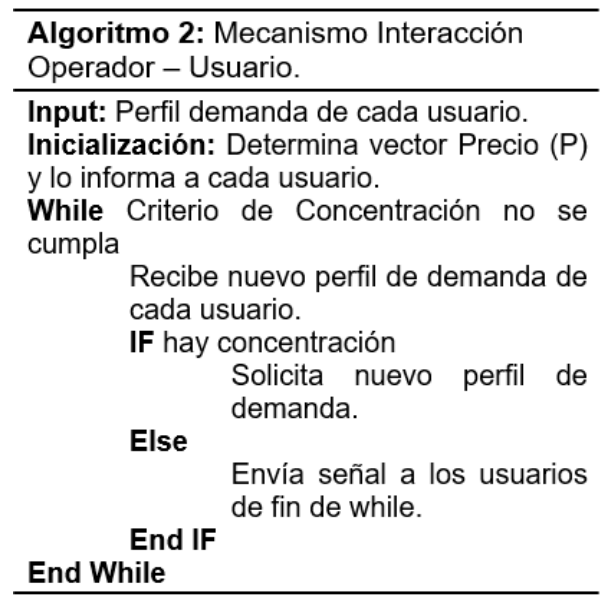

Fig. 6: Mecanismo de interacción

Para el caso propuesto, se establecen las matrices A para el usuario 1 y para el usuario 2 en el programa de Matlab, como se observa en las ecuaciones 6 y 7 respectivamente. La columna 11 remarcada pertenece a la carga flexible que es encendida en 3 horarios distintos. Esta carga es la que modificará el algoritmo genético establecido en Matlab, cuya energía es 182 Wh, como se presenta en la columna 11, que esta remarcada, en 
la ecuación 8. Se aclara que, para una mejor organización del artículo, el vector columna $E$ ha sido transpuesto en la ecuación 8 . Tanto las matrices $A$ de ambos consumidores como el vector de energía son los usados en Celiz et al. (2018)

$$
\begin{aligned}
& \begin{array}{lllllllllllllllllllllllllllll}
1 & 1 & 0 & 0 & 0 & 0 & 0 & 0 & 0 & 0 & 0 & 0 & 0 & 0 & 0 & 0 & 0 & 0 & 0 & 0 & 0 & 0 & 0 & 1 & 0 & 0 & 0 & 0
\end{array} \\
& \begin{array}{llllllllllllllllllllllllllll}
1 & 1 & 0 & 0 & 0 & 0 & 0 & 0 & 0 & 0 & 0 & 0 & 0 & 0 & 0 & 0 & 0 & 0 & 0 & 0 & 0 & 0 & 0 & 1 & 0 & 0 & 0 & 0
\end{array} \\
& \begin{array}{llllllllllllllllllllllllllll}
1 & 1 & 0 & 0 & 0 & 0 & 0 & 0 & 0 & 0 & 0 & 0 & 0 & 0 & 0 & 0 & 0 & 0 & 0 & 0 & 0 & 0 & 0 & 1 & 0 & 0 & 0 & 0
\end{array} \\
& \begin{array}{lllllllllllllllllllllllllllll}
1 & 1 & 0 & 0 & 0 & 0 & 0 & 0 & 0 & 0 & 0 & 0 & 0 & 0 & 0 & 0 & 0 & 0 & 0 & 0 & 0 & 0 & 0 & 1 & 0 & 0 & 0 & 0
\end{array} \\
& \begin{array}{llllllllllllllllllllllllllll}
1 & 1 & 0 & 0 & 0 & 0 & 0 & 0 & 0 & 0 & 0 & 0 & 0 & 0 & 0 & 0 & 0 & 0 & 0 & 0 & 0 & 0 & 0 & 1 & 0 & 0 & 0 & 0
\end{array} \\
& \begin{array}{lllllllllllllllllllllllllllll}
1 & 1 & 0 & 0 & 0 & 0 & 0 & 0 & 0 & 0 & 0 & 0 & 0 & 0 & 0 & 0 & 0 & 0 & 0 & 0 & 0 & 0 & 0 & 1 & 0 & 0 & 0 & 0
\end{array} \\
& \begin{array}{llllllllllllllllllllllllllll}
1 & 1 & 0 & 0 & 0 & 0 & 0 & 0 & 0 & 0 & 0 & 0 & 0 & 0 & 0 & 0 & 0 & 0 & 0 & 0 & 0 & 0 & 0 & 1 & 0 & 0 & 0 & 0
\end{array} \\
& \begin{array}{lllllllllllllllllllllllllllll}
1 & 1 & 0 & 0 & 0 & 0 & 0 & 0 & 0 & 0 & 0 & 0 & 0 & 0 & 0 & 0 & 0 & 0 & 0 & 0 & 0 & 0 & 0 & 1 & 0 & 1 & 0 & 0
\end{array} \\
& \begin{array}{llllllllllllllllllllllllllll}
1 & 1 & 1 & 0 & 0 & 0 & 0 & 0 & 0 & 0 & 0 & 0 & 0 & 0 & 0 & 0 & 0 & 0 & 0 & 0 & 0 & 0 & 0 & 1 & 0 & 0 & 0 & 1
\end{array} \\
& \begin{array}{llllllllllllllllllllllllllll}
1 & 1 & 0 & 0 & 0 & 0 & 0 & 0 & 0 & 0 & 0 & 0 & 0 & 0 & 0 & 0 & 0 & 0 & 0 & 0 & 0 & 0 & 0 & 1 & 0 & 0 & 0 & 0
\end{array} \\
& \begin{array}{llllllllllllllllllllllllllll}
1 & 1 & 0 & 0 & 0 & 0 & 0 & 0 & 0 & 0 & 0 & 0 & 0 & 0 & 0 & 0 & 0 & 0 & 0 & 0 & 0 & 0 & 0 & 1 & 0 & 0 & 0 & 0
\end{array} \\
& \mathrm{~A}_{\mathrm{U} 1}=\begin{array}{lllllllllllllllllllllllllllll}
1 & 1 & 0 & 0 & 0 & 0 & 0 & 1 & 0 & 0 & 0 & 0 & 0 & 0 & 0 & 0 & 0 & 0 & 0 & 0 & 0 & 0 & 0 & 1 & 0 & 0 & 0 & 0 \\
1 & 1 & 0 & 0 & 0 & 0 & 0 & 1 & 1 & 0 & 0 & 0 & 0 & 0 & 0 & 0 & 1 & 0 & 0 & 0 & 0 & 0 & 0 & 1 & 0 & 0 & 0 & 0
\end{array} \\
& \begin{array}{lllllllllllllllllllllllllllll}
1 & 1 & 0 & 0 & 0 & 0 & 0 & 1 & 1 & 0 & 0 & 0 & 0 & 0 & 0 & 0 & 1 & 0 & 0 & 0 & 0 & 0 & 0 & 1 & 0 & 0 & 0 & 0
\end{array} \\
& \begin{array}{llllllllllllllllllllllllllll}
1 & 1 & 0 & 0 & 0 & 0 & 0 & 0 & 1 & 0 & 0 & 0 & 0 & 0 & 0 & 0 & 1 & 0 & 0 & 0 & 0 & 0 & 0 & 1 & 0 & 0 & 0 & 0
\end{array} \\
& \begin{array}{llllllllllllllllllllllllllll}
1 & 1 & 0 & 0 & 1 & 1 & 0 & 0 & 1 & 0 & 1 & 0 & 0 & 0 & 0 & 0 & 1 & 0 & 0 & 0 & 0 & 0 & 1 & 1 & 0 & 0 & 1 & 0
\end{array} \\
& \begin{array}{lllllllllllllllllllllllllllll}
1 & 1 & 0 & 0 & 1 & 1 & 0 & 0 & 0 & 0 & 0 & 0 & 0 & 0 & 0 & 0 & 1 & 0 & 0 & 0 & 0 & 0 & 0 & 1 & 0 & 0 & 0 & 0
\end{array} \\
& \begin{array}{lllllllllllllllllllllllllllll}
1 & 1 & 1 & 0 & 0 & 0 & 0 & 0 & 0 & 0 & 0 & 0 & 0 & 0 & 0 & 0 & 1 & 0 & 0 & 0 & 0 & 0 & 0 & 1 & 0 & 0 & 0 & 0
\end{array} \\
& \begin{array}{llllllllllllllllllllllllllll}
1 & 1 & 0 & 1 & 0 & 0 & 0 & 0 & 0 & 0 & 1 & 0 & 0 & 0 & 0 & 1 & 1 & 0 & 0 & 0 & 1 & 0 & 0 & 1 & 0 & 0 & 0 & 0
\end{array} \\
& \begin{array}{llllllllllllllllllllllllllll}
1 & 1 & 0 & 0 & 0 & 0 & 0 & 0 & 1 & 0 & 0 & 0 & 0 & 0 & 0 & 1 & 1 & 0 & 0 & 0 & 0 & 0 & 0 & 1 & 0 & 0 & 0 & 0
\end{array} \\
& \begin{array}{llllllllllllllllllllllllllll}
1 & 1 & 0 & 0 & 0 & 0 & 0 & 1 & 1 & 0 & 0 & 0 & 0 & 0 & 0 & 1 & 0 & 0 & 0 & 0 & 0 & 0 & 0 & 1 & 0 & 0 & 0 & 0
\end{array} \\
& \begin{array}{llllllllllllllllllllllllllll}
1 & 1 & 1 & 0 & 1 & 1 & 1 & 1 & 1 & 0 & 1 & 1 & 1 & 0 & 0 & 0 & 0 & 0 & 0 & 0 & 0 & 0 & 0 & 1 & 0 & 0 & 0 & 0
\end{array} \\
& \begin{array}{llllllllllllllllllllllllllll}
1 & 1 & 0 & 0 & 1 & 1 & 1 & 0 & 1 & 1 & 0 & 1 & 1 & 0 & 0 & 0 & 0 & 0 & 0 & 0 & 0 & 0 & 0 & 1 & 0 & 0 & 0 & 0
\end{array} \\
& \begin{array}{lllllllllllllllllllllllllllll}
1 & 1 & 0 & 0 & 1 & 1 & 1 & 0 & 0 & 1 & 0 & 1 & 1 & 0 & 0 & 0 & 0 & 0 & 0 & 0 & 0 & 0 & 1 & 1 & 0 & 0 & 0 & 0
\end{array} \\
& \begin{array}{llllllllllllllllllllllllllll}
1 & 1 & 0 & 0 & 1 & 1 & 1 & 0 & 0 & 0 & 0 & 1 & 1 & 0 & 0 & 0 & 0 & 0 & 0 & 0 & 0 & 0 & 0 & 1 & 0 & 0 & 1 & 0
\end{array} \\
& \begin{array}{llllllllllllllllllllllllllll}
1 & 1 & 0 & 0 & 0 & 0 & 0 & 0 & 0 & 0 & 0 & 0 & 0 & 0 & 0 & 0 & 0 & 0 & 0 & 0 & 0 & 0 & 0 & 1 & 0 & 0 & 0 & 0
\end{array} \\
& \begin{array}{llllllllllllllllllllllllllll}
1 & 1 & 0 & 0 & 0 & 0 & 0 & 0 & 0 & 0 & 0 & 0 & 0 & 0 & 0 & 0 & 0 & 0 & 0 & 0 & 0 & 0 & 0 & 1 & 0 & 0 & 0 & 0
\end{array} \\
& \begin{array}{llllllllllllllllllllllllllll}
1 & 1 & 0 & 0 & 0 & 0 & 0 & 0 & 0 & 0 & 0 & 0 & 0 & 0 & 0 & 0 & 0 & 0 & 0 & 0 & 0 & 0 & 0 & 1 & 0 & 0 & 0 & 0
\end{array} \\
& \begin{array}{llllllllllllllllllllllllllll}
1 & 1 & 0 & 0 & 0 & 0 & 0 & 0 & 0 & 0 & 0 & 0 & 0 & 0 & 0 & 0 & 0 & 0 & 0 & 0 & 0 & 0 & 0 & 1 & 0 & 0 & 0 & 0
\end{array} \\
& \begin{array}{lllllllllllllllllllllllllllll}
1 & 1 & 0 & 0 & 0 & 0 & 0 & 0 & 0 & 0 & 0 & 0 & 0 & 0 & 0 & 0 & 0 & 0 & 0 & 0 & 0 & 0 & 0 & 1 & 0 & 0 & 0 & 0
\end{array} \\
& \begin{array}{llllllllllllllllllllllllllll}
1 & 1 & 0 & 0 & 0 & 0 & 0 & 0 & 0 & 0 & 0 & 0 & 0 & 0 & 0 & 0 & 0 & 0 & 0 & 0 & 0 & 0 & 0 & 1 & 0 & 0 & 0 & 0
\end{array} \\
& \begin{array}{llllllllllllllllllllllllllll}
1 & 1 & 0 & 0 & 0 & 0 & 0 & 0 & 0 & 0 & 0 & 0 & 0 & 0 & 0 & 0 & 0 & 0 & 0 & 0 & 0 & 0 & 0 & 1 & 0 & 0 & 0 & 0
\end{array} \\
& \begin{array}{llllllllllllllllllllllllllll}
1 & 1 & 0 & 0 & 0 & 0 & 0 & 0 & 0 & 0 & 0 & 0 & 0 & 0 & 0 & 0 & 0 & 1 & 0 & 0 & 0 & 0 & 0 & 1 & 0 & 0 & 0 & 0
\end{array} \\
& \begin{array}{llllllllllllllllllllllllllll}
1 & 1 & 1 & 0 & 0 & 0 & 0 & 0 & 0 & 0 & 1 & 0 & 0 & 0 & 0 & 0 & 0 & 1 & 0 & 0 & 0 & 0 & 0 & 1 & 0 & 0 & 0 & 0
\end{array} \\
& \begin{array}{llllllllllllllllllllllllllll}
1 & 1 & 0 & 0 & 0 & 0 & 0 & 1 & 0 & 0 & 1 & 0 & 0 & 0 & 0 & 0 & 0 & 1 & 0 & 0 & 0 & 0 & 0 & 1 & 0 & 0 & 0 & 0
\end{array} \\
& \begin{array}{lllllllllllllllllllllllllllll}
1 & 1 & 0 & 0 & 0 & 0 & 0 & 1 & 0 & 0 & 0 & 0 & 0 & 0 & 0 & 0 & 0 & 0 & 0 & 0 & 0 & 0 & 0 & 1 & 0 & 0 & 0 & 0
\end{array} \\
& A_{\cup 2}=\begin{array}{lllllllllllllllllllllllllllll}
1 & 1 & 0 & 0 & 0 & 0 & 0 & 0 & 0 & 0 & 0 & 0 & 0 & 0 & 0 & 1 & 0 & 0 & 0 & 0 & 0 & 0 & 0 & 1 & 0 & 0 & 0 & 0 \\
1 & 1 & 0 & 0 & 1 & 1 & 0 & 0 & 0 & 1 & 0 & 0 & 0 & 0 & 0 & 1 & 0 & 0 & 0 & 0 & 0 & 0 & 0 & 1 & 0 & 0 & 0 & 0
\end{array} \\
& \begin{array}{lllllllllllllllllllllllllllll}
1 & 1 & 0 & 0 & 1 & 1 & 0 & 0 & 0 & 1 & 0 & 0 & 0 & 0 & 0 & 1 & 0 & 0 & 0 & 0 & 0 & 0 & 0 & 1 & 0 & 0 & 0 & 0
\end{array} \\
& \begin{array}{llllllllllllllllllllllllllll}
1 & 1 & 0 & 0 & 1 & 1 & 0 & 0 & 0 & 1 & 0 & 0 & 0 & 0 & 0 & 0 & 0 & 0 & 0 & 0 & 0 & 0 & 0 & 1 & 0 & 0 & 0 & 0
\end{array} \\
& \begin{array}{llllllllllllllllllllllllllll}
1 & 1 & 0 & 0 & 1 & 1 & 0 & 1 & 0 & 0 & 0 & 0 & 0 & 0 & 0 & 0 & 0 & 0 & 0 & 0 & 0 & 0 & 0 & 1 & 0 & 0 & 0 & 0
\end{array} \\
& \begin{array}{llllllllllllllllllllllllllll}
1 & 1 & 0 & 0 & 0 & 0 & 0 & 1 & 0 & 0 & 0 & 0 & 0 & 0 & 0 & 0 & 0 & 0 & 0 & 0 & 0 & 0 & 0 & 1 & 0 & 0 & 0 & 0
\end{array} \\
& \begin{array}{lllllllllllllllllllllllllllll}
1 & 1 & 1 & 0 & 0 & 0 & 0 & 1 & 0 & 0 & 0 & 0 & 0 & 0 & 0 & 0 & 0 & 0 & 0 & 0 & 0 & 0 & 0 & 1 & 0 & 0 & 0 & 0
\end{array} \\
& \begin{array}{llllllllllllllllllllllllllll}
1 & 1 & 0 & 0 & 0 & 0 & 0 & 1 & 0 & 0 & 0 & 0 & 0 & 0 & 0 & 0 & 0 & 0 & 0 & 0 & 0 & 0 & 0 & 1 & 0 & 0 & 0 & 0
\end{array} \\
& \begin{array}{lllllllllllllllllllllllllllll}
1 & 1 & 0 & 0 & 0 & 0 & 1 & 1 & 0 & 0 & 0 & 0 & 0 & 0 & 0 & 0 & 0 & 0 & 0 & 0 & 0 & 0 & 1 & 1 & 0 & 0 & 0 & 0
\end{array} \\
& \begin{array}{llllllllllllllllllllllllllll}
1 & 1 & 0 & 1 & 1 & 1 & 1 & 0 & 0 & 0 & 0 & 0 & 0 & 0 & 0 & 0 & 0 & 1 & 0 & 0 & 0 & 1 & 0 & 1 & 0 & 0 & 0 & 0
\end{array} \\
& \begin{array}{llllllllllllllllllllllllllll}
1 & 1 & 1 & 0 & 1 & 1 & 1 & 0 & 0 & 0 & 0 & 0 & 0 & 0 & 0 & 0 & 0 & 1 & 0 & 0 & 0 & 0 & 0 & 1 & 0 & 0 & 0 & 0
\end{array} \\
& \begin{array}{llllllllllllllllllllllllllll}
1 & 1 & 0 & 0 & 1 & 1 & 1 & 0 & 0 & 0 & 0 & 0 & 0 & 0 & 0 & 0 & 0 & 1 & 0 & 0 & 0 & 0 & 0 & 1 & 0 & 0 & 0 & 0
\end{array} \\
& \begin{array}{llllllllllllllllllllllllllll}
1 & 1 & 0 & 0 & 1 & 1 & 1 & 0 & 0 & 0 & 0 & 0 & 0 & 0 & 0 & 0 & 0 & 1 & 0 & 0 & 0 & 0 & 0 & 1 & 0 & 0 & 0 & 0
\end{array} \\
& \begin{array}{llllllllllllllllllllllllllll}
1 & 1 & 0 & 0 & 1 & 1 & 1 & 0 & 0 & 0 & 0 & 0 & 0 & 0 & 0 & 0 & 0 & 1 & 0 & 0 & 0 & 0 & 0 & 1 & 0 & 0 & 1 & 0
\end{array} \\
& E^{\prime}=[981016760010043,410260901418210043,425053100101312006065025100900906751201050225]
\end{aligned}
$$

La ecuación 9 es la utilizada en el algoritmo programado en Matlab para minimizar el costo del consumidor 1, análogamente se obtiene la ecuación para el usuario 2

$$
\min C_{u 1}=\mathrm{P}^{\prime} \cdot A_{u 1}(t) \cdot E
$$

Donde t representa la columna correspondiente a la carga flexible. Para ello, el GA dispone de tres variables $\left(u_{1}, u_{2} y u_{3}\right)$ que indican el horario de encendido en cada carga de tal modo que se obtiene el vector $U_{b}=\left[u_{1}\right.$, $\left.\mathrm{u}_{2}, \mathrm{u}_{3}\right]$. Entonces el GA modifica Ub para minimizar C. El código usado que modifica $A_{\mathrm{u} 1}$ es:

$\mathrm{t}=\operatorname{zeros}(24,1)$;

$t\left(U b_{u 1}, 1\right)=$ encendido; 


$$
A_{u 1}(1: 24,[11])=t
$$

Se utiliza Ub 1 para el usuario 1 , de forma similar se procede con el consumidor 2 . La distribuidora, en caso de observar que Ubu1 es igual a Ubu2 pedirá que vuelvan a proponer una nueva optimización de sus cargas.

De la multiplicación de (6) y (8) se obtiene el vector Xu1 para el usuario 1 y análogamente con (7) y (8) se obtiene $X_{\mathrm{u} 2}$. Para una mejor visualización se presenta la suma de $\mathrm{X}_{\mathrm{u} 1}$ con $\mathrm{X}_{\mathrm{u} 2}$ en las Figuras 7,8 y 9 . El rojo en cada imagen representa las cargas no interrumpibles ni desplazables y en azul las cargas interrumpibles o desplazables, ambas en kWh. La línea punteada representa la tarifa horaria, este esquema también se puede observar en ecuación 10. 1) Se parte de la curva de demanda de ambos usuarios en el escenario tradicional (Fig. 7); 2) la distribuidora propone el sistema tarifario P durante el día (Fig. 7, línea punteada); 3) cada jugador elabora su estrategia de juego y se la informa a la distribuidora (Fig. 8); 4) si la distribuidora determina que hay acumulación de cargas en un horario y que estas son flexibles para ambos usuarios, les solicita una nueva estrategia de consumo. (Fig. 9).

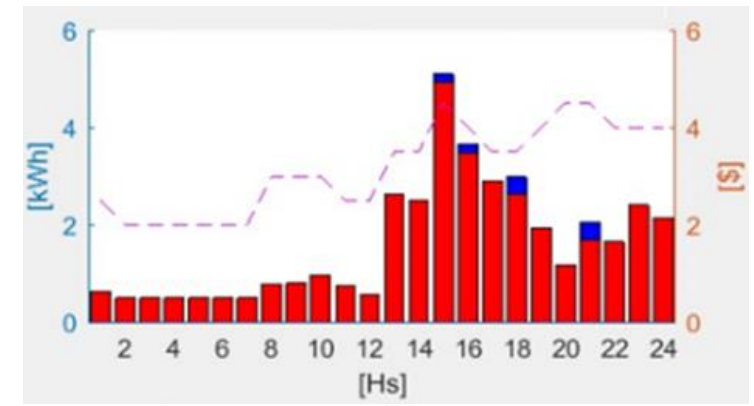

Fig. 7: Energía Consumida por dos usuarios sin optimización

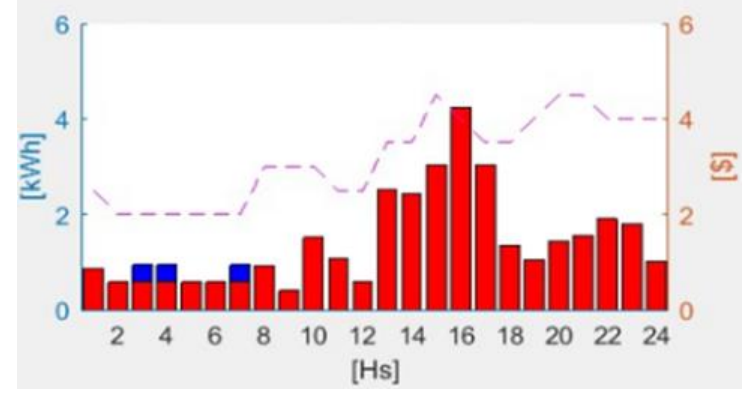

Fig. 8: energía consumida por dos usuarios con optimización en primera ronda

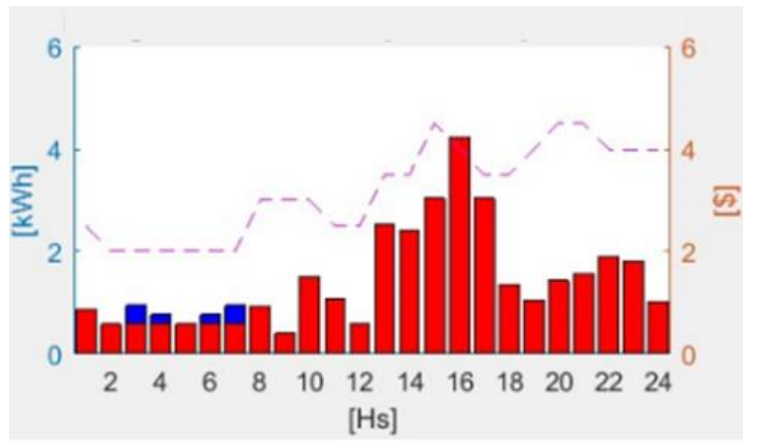

Fig. 9: energía consumida por dos usuarios con optimización en segunda ronda

$\mathrm{P}=\left[\begin{array}{llllllllllllllllllllllll}2,5 & 2 & 2 & 2 & 2 & 2 & 2 & 3 & 3 & 3 & 2,5 & 2,5 & 3,5 & 3,5 & 4,5 & 4 & 3,5 & 3,5 & 4 & 4,5 & 4,5 & 4 & 4 & 4\end{array}\right]$

En la Figura 8 se observa que ambos usuarios eligieron los mismos horarios para desplazar su carga, o sea hay coincidencia en los horarios 3, 4 y 7; mientras que en la Figura 9, que es el resultado de la segunda ronda se observa que solo coinciden en los horarios 3 y 7 . El paso cuatro muestra la ventaja de este control. Además, como se observa en Tabla 2, el costo disminuye al optimizar el consumo y en este caso se mantiene constante tanto en ronda 1 como en ronda 2 mientras se logra una mejor distribución de la carga.

Tabla 2: Costo de la energía consumida por usuario

\begin{tabular}{cccc}
\hline Usuario & $\begin{array}{c}\text { Costo } \\
\text { sin optimizar }\end{array}$ & $\begin{array}{c}\text { Costo } \\
\text { ronda 1 }\end{array}$ & $\begin{array}{c}\text { Costo } \\
\text { ronda 2 }\end{array}$ \\
\hline Usuario 1 & 78,213 & 77,030 & 77,030 \\
\hline Usuario 2 & 75,428 & 74,335 & 74,335 \\
\hline
\end{tabular}

\section{CONCLUSIONES}

Ante un esquema de optimización en la demanda de la red, el distribuidor necesita que los usuarios modifiquen sus cargas de manera coordinada para evitar un nuevo pico de demanda, como esto afecta la privacidad de los usuarios o implica una comunicación entre los usurarios es un escenario difícil de aplicarse. Por ello se propone un control indirecto hibrido que implica una comunicación con información restringida entre los usuarios y el 
distribuidor. Para el caso propuesto el operador conoce la modificación de las cargas flexibles de los dos usuarios planteados y tiene como objetivo aplanar la curva de la demanda de la red, pero es el usuario quien tiene el control y acción sobre su demanda. A partir de la interacción usuario-distribuidor fue posible disminuir la concentración y aplanar la curva de demanda de la red. Esto se lo logró afectando mínimamente la privacidad de los usuarios. Por ello este esquema propuesto será posible aplicarlo a los usuarios residenciales.

Se opto por el algoritmo genético al ser una buena herramienta de optimización para este tipo de problemas ya que su naturaleza aleatoria permite que, ante el pedido del distribuidor de volver a realizar la optimización de la demanda, las cargas de los dos usuarios se desplacen a otros puntos óptimos, evitando la concentración. Un algoritmo tradicional, al no tener naturaleza aleatoria, tendría la desventaja de dar el mismo resultado ante el pedido del distribuidor de realizar nuevamente la optimización.

Este enfoque es novedoso ya que se centra en la interacción usuario-distribuidor, dejando la optimización a cargo del usuario pero, permitiendo la intervención de la distribuidora por medio de la interacción, solicitando al usuario una nueva propuesta de optimización, si se lo considera necesario.

\section{REFERENCIAS}

Belhaiza, S. y Baroudi, U., A Game Theoretic Model for Smart Grids Demand Management, https://doi.org/10.1109/TSG.2014.2376632, IEEE Transactions on Smart Grid, 6(3), 1386-1393 (2014).

Bian, D., Pipattanasomporn, M. y Rahman, S., A Human Expert-based Approach to Electrical Peak Demand Management, https://doi.org/10.1109/PESGM.2015.7286112, IEEE Transactions on Power Delivery, 30(3), 1119-1127 (2015).

Blaauwbroek, N., Nguyen, P. y Slootweg, H., Applying Demand Side Management Using a Generalised Three Phase Grid Supportive Approach, https://doi.org/10.1109/EEEIC.2017.7977412, 2017 IEEE International Conference on Environment and Electrical Engineering and 2017 IEEE Industrial and Commercial Power Systems Europe (EEEIC/l\&CPS Europe), 16, IEEE (2017).

Celiz, D. H., Figueroa Etchecopar, M. y otros cuatro autores, Estudio y Análisis para Definir Políticas que Modifiquen las Conductas de Consumo en Usuarios Domiciliarios Monofásicos, IV Congreso Argentino de Ingeniería - X Congreso Argentino de Enseñanza de la Ingeniería (CADI-CAEDI 2018), Córdoba, Argentina (2018).

Dethlefs, T., Preisler, T. y Renz, W., Multi-agent-based distributed optimization for demand-side-management applications, https://doi.org/10.15439/2014F251, 2014 Federated Conference on Computer Science and Information Systems, 14891496, IEEE (2014).

Duman, A. C., Güler, Ö., Deveci, K. y Gönül, Ö., Residential Load Scheduling Optimization for Demand-side Management under Time-of-Use Rate., https://doi.org/10.1109/SGCF.2018.8408971, 2018 6th International Istanbul Smart Grids and Cities Congress and Fair (ICSG), 193-196, IEEE (2018).

ENRE, Consumo indicativo de algunos artefactos eléctricos. En la web: https://www.enre.gov.ar/web/web.nsf/Consumo? OpenPage, acceso 10 de noviembre (2019)

Erdinc, O., Taşcıkaraoğlu, A. y otros tres autores, End-user Comfort Oriented Day-ahead Planning for Responsive Residential HVAC Demand Aggregation Considering Weather Forecasts, https://doi.org/10.1109/TSG.2016.2556619, IEEE Transactions on Smart Grid, 8(1), 362-372 (2016).

Gupta, I., Anandini, G. y Gupta, M., An Hour Wise Device Scheduling Approach for Demand Side Management in Smart Grid using Particle Swarm Optimization, https://doi.org/10.1109/NPSC.2016.7858965, 2016 National Power Systems Conference (NPSC), 1-6, IEEE (2016).

Huang, G., Yang, J. y Wei, C., Cost-Effective and Comfort-aware Electricity Scheduling for Home Energy Management System, https://doi.org/10.1109/BDCloud-SocialCom-SustainCom.2016.73, 2016 IEEE International Conferences on Big Data and Cloud Computing (BDCloud), Social Computing and Networking (SocialCom), Sustainable Computing and Communications (SustainCom) (BDCloud-SocialCom-SustainCom), 453-460, IEEE (2016).

INTI, Eficiencia energética en uso residencial. En la web: https://www.editores-srl.com.ar/revistas/ac/10/inti_eficiencia_e nergetica, acceso 10 de noviembre (2019)

Janocha, S., Baum, S. y Stadler, I., Cost Minimization by Optimization of Electricity Generation and Demand Side Management, https://doi.org/10.1109/IESC.2016.7569489, 2016 International Energy and Sustainability Conference (IESC), 1-7, IEEE (2016).

Jayadev, V. y Swarup, K. S., Optimization of Microgrid with Demand Side Management using Genetic Algorithm, https://doi.org/10.1049/ic.2013.0124, IET Conference on Power in Unity: a Whole System Approach, 1-12 (2013).

Kassakian, J. G., Schmalensee, R. y otros veintitres autores, The Future of the Electric Grid: An Interdisciplinary MIT Study, URL http://energy.mit.edu/research/future-electric-grid/, [Accessed 16 Aug 2018] (2011).

Lin, S. K. y Chen, C. R., Optimal Energy Consumption Scheduling in Home Energy Management System, https://doi.org/10.1109/ICMLC.2016.7872962, 2016 International Conference on Machine Learning and Cybernetics (ICMLC), 2, 638-643, IEEE (2016). 
Gestal, M., Rivero, D. y otros tres autores, Introducción a los Algoritmos Genéticos y la Programación Genética, 1-76, Universidade da Coruña, Servizo de Publicacións, La Coruña, España (2010).

Melhem, F. Y., Grunder, O., Hammoudan, Z. y Moubayed, N., Energy Management in Electrical Smart Grid Environment using Robust Optimization Algorithm, https://doi.org/10.1109/TIA.2018.2803728, IEEE Transactions on Industry Applications, 54(3), 2714-2726 (2018).

Pavithra, N. y Esther, B. P., Residential Demand Response using Genetic Algorithm. https://doi.org/10.1109/IPACT.2017.8245143, 2017 Innovations in Power and Advanced Computing Technologies (iPACT), 1-4. IEEE (2017).

Rahate, N. D. y Kinhekar, N., Demand Side Management for Household Equipment's, https://doi.org/10.1109/ICOMICON.2017.8279108, 2017 International Conference on Information, Communication, Instrumentation and Control (ICICIC), 1-5, IEEE (2017).

Restrepo, Á. R., Nope, S. E. y Enríquez, D. E., Beneficios Económicos de la Gestión de la Demanda y la Energía Autogenerada en el Contexto de la Regulación Colombiana. Información tecnológica, 29(1), 105-116. (2018)

Seema, P. N., Deepa, V. y Nair, M. G., Consumer Level Intelligence in a Smart Micro-Grid, https://doi.org/ 10.1109/ICEETS.2016.7583773, 2016 International Conference on Energy Efficient Technologies for Sustainability (ICEETS), 320-324, IEEE (2016).

Shao, S., Pipattanasomporn, M. y Rahman, S., An Approach for Demand Response to Alleviate Power System Stress Conditions, https://doi.org/10.1109/PES.2011.6039852, 2011 IEEE Power and Energy Society General Meeting, 1-7. IEEE (2011).

Silva, A., Marinheiro, J., Cardoso, H. L. y Oliveira, E., Demand-Side Management in Power Grids: An Ant Colony Optimization Approach, https://doi.org/10.1109/CSE.2015.31, 2015 IEEE 18th International Conference on Computational Science and Engineering, 300-306, IEEE (2015)

Suryanarayanan, S., Mancilla-David, F., Mitra, J. y Li, Y., Achieving the Smart Grid through Customer-driven Microgrids Supported by Energy Storage, https://doi.org/10.1109/ICIT.2010.5472581, 2010 IEEE International Conference on Industrial Technology, 884-890, IEEE (2010).

Tan, W. N., Gan, M. T. y Tan, Z. L., Optimization Models for Demand-side and Supply-side Scheduling in Smart Grids, https://doi.org/10.1109/EEEIC.2016.7555476, 2016 IEEE 16th International Conference on Environment and Electrical Engineering (EEEIC), 1-5, IEEE (2016).

Tatarenko, T. y Garcia-Moreno, L., A Game Theoretic and Control Theoretic Approach to Incentive-based Demand Management in Smart Grids, https://doi.org/10.1109/MED.2014.6961444, 22nd Mediterranean Conference on Control and Automation, 634-639, IEEE (2014).

Vidal, A. R., Jacobs, L. A. y Batista, L. S., An Evolutionary Approach for the Demand Side Management Optimization in Smart Grid, https://doi.org/10.1109/CIASG.2014.7011561, 2014 IEEE Symposium on Computational Intelligence Applications in Smart Grid (CIASG), 1-7, IEEE (2014).

Wang, F., Zhou, L., y otros cinco autores, Multi-objective Optimization Model of Source-load-storage Synergetic Dispatch for a Building Energy Management System Based on TOU Price Demand Response, https://doi.org/10.1109/TIA.2017.2781639, IEEE Transactions on Industry Applications, 54(2), 1017-1028 (2018).

Wang, Y., Hao, C. y Yoshimura, T., A Particle Swarm Optimization and Branch and Bound Based Algorithm for Economical Smart Home Scheduling, https://doi.org/10.1109/MWSCAS.2017.8052898, 2017 IEEE 60th International Midwest Symposium on Circuits and Systems (MWSCAS), 213-216, IEEE (2017).

Zafar, A., Shah, S. y otros cuatro autores, A Meta-heuristic Home Energy Management System, https://doi.org/10.1109/WAINA.2017.118, 2017 31st International Conference on Advanced Information Networking and Applications Workshops (WAINA), 244-250, IEEE (2017).

Zhu, Z., Lambotharan, S., Chin, W. H. y Fan, Z., A Game Theoretic Optimization Framework for Home Demand Management Incorporating Local Energy Resources, https://doi.org/10.1109/TII.2015.2390035, IEEE Transactions on Industrial Informatics, 11(2), 353-362 (2015). 
